\title{
LABOUR MARKET, FOREIGN EMPLOYMENT AND REMITTANCE IN NEPAL
}

\author{
Keshav Bhakta Sapkota*
}

\section{ABSTRACT}

Foreign employment is an important part of Nepalese labour market. The national poverty reduction strategy and the previous plans have given priority to foreign employment as a source of labour absorption and poverty alleviation. This study focuses on Nepalese labour market condition, foreign employment, remittance inflow and their contribution on national economy. In Nepal, foreign employment and remittance inflow have been issues of concern over the last two decades. In this connection foreign employment was estimated to be 4.36 million up to the fiscal year 2016/17 (except India) and 56 percent households had received remittances in the fiscal year 2010/11.

Key words: Labour, market, employment, skilled, remittance, migration.

\section{INTRODUCTION AND OBJECTIVE}

According to latestcensus, thepopulationof Nepalwasapproximately 26.5 million in 2011. The average population of Nepal between 2001 and 2011 was 1.35 percent observed in the previous decade. There were some sort of positive trends observed in the population characteristics of Nepal. The average household size has declined from 5.44 in 2001 to 4.88 in 2011. Fertility rate has declined rapidly and the dependency ratio, albeit high in a decreasing trend (CBS, 2012). The rapidly declining trend of dependency ratio has also opened an avenue to the advantages of 'demographic dividend'. This trend, not only increases labour supply but also helps to increase output and saving. Urbanization speed is also seemed to be increased rapidly in the country. These changes have provoked to expland the productive base of the economy considerably.

The other side of the demographic trend, however, is a challenge for Nepal. The population growth rates of the past have produced higher number of people in the younger age categories. This trend coupled with less expected job creating opportunity in the country has resulted in higher

* Dr. Sapkota is an Associate Professor, Birendra Campus, Chitwan, TU. 
level of underemployment and low labor productivity (Pyakuryal, 2015). Nepal Labour Force Survey (2008) shows that young people from 15 and 24 years were relatively faced with sharply escalating unemployment. The massive outflow of young and productive work force has limited the scope for raising country's economic growth rate, despite the fact that Nepal has one of the highest rates of labour force growth in the Asia and the Pacific region.

Excessive dependence on remittance might not be sustainable given the fragile state of national and international economics. The 'remittance cushion' will further create a vicious cycle of high remittance and the loss of productive workforce in the economy (Pyakuryal, 2013). If Nepal could strategically and systematically manage the foreign employment process and remittance transfer, it would have collected more revenues from remittances. Many Nepalese migrants have been forced to work in poor conditions, get lower wages with high cost of living in their destinations, were victims of fraud recruitment and lack of social protection. (Karna, 2017).

In spite of growing trade deficit, surplus position in current account and BOP is due to elevated level of remittance inflow in recent years. Nepal were in fact exporting labour and receiving remittance. Excluding remittance coming from India, which was the traditional destination for many Nepalese people to seek for employment, remittance from other countries has been increasing significantly. With the escalation of internal conflict, easy issuance of passport even from district offices, and official encouragement for foreign employment which was lucrative for Nepalese people, outflows of Nepalese migrant workers increased. As a result, in terms of GDP, remittance inflow, which was about 0.70 percent during 1975-1990 and just 0.50 percent during 1990-1995, increased to 21.41 percent of GDP during 2009-2015 (Shrestha, 2017).

Meaningful use of remittances at macro level boots macro activities which ultimately could raise socio-economic status. Remittance could play an important role to reduce poverty and sustain the desired level of development goal set by country. Additionally, it could reduce the dependency on foreign debt and aid too. Income unfortunately have been found to be used for unproductive purpose at micro level. Some 80 percent 
of this income have been found to be spent on house construction, buying land, meeting household expenses, etc. Some 20 percent remittances have found to be used for education for children, running business, cultivating farm land etc. It indicates that Nepal still lags on the meaningful productive uses of remittances money for the betterment of the people and economy as a whole. The aim of this paper is to show current status of foreign employment and remittance inflow and analyze the contribution of remittance inflow on poverty reduction strategy of Nepal.

\section{METHODOLOGY}

The research design followed in this study is analytical as well as descriptive. It is based on secondary data. Relevant data published from Central Bureau of statistics (CBS), Nepal Rastra Bank, Ministry of Finance Department of Foreign Employment (DoFE) and other concerned institutions has been taken into consideration.

\section{DISCUSSION AND RESULT}

\section{Labour Market and Situation of Employment}

Nepal's labour force was rapidly growing. More than 450 thousand youths enter the labour market annually (NPC, 2017). The main reasons for rapid growth of labour force were the faster increase in population and raising proportion of women entering into the labour market (Pyakuryal, 2015). The fast growth of labour force places a great strain on the ability of the economy to generate additional work opportunities on a sufficient scale to absorb the rising numbers. This was mainly because of inadequate agricultural based economy growth and slow transformation of agricultural based economy to modern economy. In addition, the country suffered from armed conflict between 1996-2006. Political instability continued even after the peace agreement was signed between the conflicting parties and the government in 2006 (Pyakuryal, 2015).

A comparison of labour productivity level in 2011, measured as GDP at constant prices per worker had indicated that Nepal's per worker productivity was the lowest in Asia. It was about two-thirds of labour productivity in Bangladesh, about one third that in India and Pakistan indicate that there was a gigantic dearth of productive employment opportunity in the country. 
Even though the population growth rate was declining in Nepal, labour force growth was not followed by declining population growth rate. Between 2008 and 2013 (CBS, 2009 and CBS, 2014) the population aged 1559 grew by an estimated rate of 2.25 percent per annum, which translates into an annual labour of $3,68.000$. The labour force participating rate is constantly increasing in Nepal. It was not only due to change in demographic features but also the changing rate of women in the country. Nepal Living Standard Survey (CBS, 2011) shows that the male labour force participation rate increased from 75 percent in 1996 to 81 percent in 2011. The participation of female labour force increased even more rapidly from 66 percent to 79 percent during the same period. The unemployment rate was very low at 3.3 percent in 2013, but was contained to increase from 1.8 percent in 1998/99. The rate of unemployment was much higher in urban areas (8\%) and among the youths. The unemployment rate at the age group 15-24 in urban areas in 2013 was 14 percent (CBS, 2014).

The unemployment rate was low in Nepal however, the underemployment was high. Time-related underemployment measured as those who worked less than 40 hours a week was as high as 56 percent in 2013. In countries, where social perfection and social safety net is still not available everybody has to find a job whether it is precariously low productive and seasonal. That was the reason why unemployment was not an option where people could survive. Approximately, 32 percent male and 55 percent female worked less than 40 hours a week in 2013 (CBS,2014). One of the primary reasons for under employment, according to the labor force survey of 2008 was mismatches among 45 percent of the labor force. The reasons for underemployment according to the same survey were seeking jobs 41 percent off season inactivity 56 percent and others 3 percent (CBS, 2009). the sectoral composition of employment was seemed to be agricultural sectors (Table 1).

Table 1: Sectoral Composition of Employment, (1998-99, 2008 and 2012-13) 


\begin{tabular}{|l|c|c|c|}
\hline \multicolumn{1}{|c|}{ sector } & $\begin{array}{c}\text { Percentage } \\
\text { of total } \\
\text { Employment } \\
\text { in 1998-99 }\end{array}$ & $\begin{array}{c}\text { Percentage } \\
\text { of total } \\
\text { employment } \\
\text { in 2008 }\end{array}$ & $\begin{array}{c}\text { Percentage } \\
\text { of total } \\
\text { employment } \\
\text { in 2013 }\end{array}$ \\
\hline $\begin{array}{l}\text { Agriculture, forestry } \\
\text { and fishing }\end{array}$ & 78.00 & 73.87 & 66.5 \\
\hline Manufacturing & 5.85 & 6.56 & 5.2 \\
\hline Construction & 3.54 & 3.19 & 5.0 \\
\hline Industry & 10.50 & 10.90 & 11 \\
\hline Total Services & 11.54 & 15.22 & 22.4 \\
\hline
\end{tabular}

Source: Islam (2014), CBS (2014).

The agriculture sector in 2013 accounted for 66.5 percent of the total employment in Nepal. Industrial sector and service sector accounted for 11 percent and 22.4 percent of the total employment respectively (Table 1). Low level of industrialization and its smaller contribution in total employment, higher agricultural employment and transformation to low -productive services indicate that the economy is still least diversified and largely traditional in terms of employment opportunities in the country. Self employment in agriculture still dominates the employment situation in Nepal. This was the main sector of employment and accounts for 61 percent of the total self- employed in the country (CBS, 2011). A higher percentage of females were self employed in agriculture and in the allied economic activities compared to their male counterparts.

The share of industry, in general, had stagnated between 1998/1999 and 2012/2013. Dismal performance of export oriented industries, rapidly rising imports, rigid labor laws, infrastructural bottlenecks, open border with India and rapidly increasing informal border trade with China, have made Nepal's manufacturing sector uncompetitive. Its share in GDP as well as employment had been declined. As a result the overall capability of industry in creating employment found to be eroded. The share of construction sector on employment also declined during 1998/1999-2008, mainly due to the intensified armed conflict, political instability and declining governments share in gross fixed capital formation. However, higher employment growth had been observed in this sector between 2008-2013, possibly due to revival 
in private as well as public construction industries (Islam, 2014, CBS, 2008 \& CBS, 2009). The main growth in employment creation had been observed in the service sector. Its share in employment increased from 11.5 percent in 1998/1999 to 22.4 percent in 2013. Increased share of services sector and stagnant share of industrial sector in employment reflects a shift of labor out-flow from low productive activities mainly in agriculture to low productive services (largely non-tradable). The service sector had accounted for a greater share of Nepal's labor force overtime, since much of the sector was composed of informal activities. Nepal's economy is still dominated by informal sector's activities. According to a World Bank study the informal economy in Nepal constitutes about 37.5 percent of the GDP, a size higher than in other countries of south Asia (World Bank, 2011). Its share in employment was estimated 96 percent. These numbers were alarming. Informal sector was known to private low equality, low productive and poorly remunerated employment opportunities. Additionally, most informal sector jobs were in rural areas (68.3 percent). It aggregates rural urban and put more people in rural areas in risk of falling into the poverty trap (Sijapati 2014). In terms of areas of work, informal employment was all pervasive. Sectors which had the highest degree of formality were the public sector, financial and transport and communication sector.

\section{Foreign Employment, Remittance and Its Use}

With the globalization process, Nepal opened foreign employment officially to cure the unemployment problem that emerged from higher growth of labour supply amidst sluggish economic growth. It was believed that the lack of employment opportunities was one of the reasons to ignite internal conflict in 1996 while Nepal was in the process of rapid integration with the world. International labour migration had emerged as an alternative livelihood opportunity for many Nepali households in recent years, although history of labour migration dates back in 300 years ago. In the past, much of labour migration from Nepal mainly concentrated to India, at least up to the mid-1980s. Then new destinations emerged with the intensification of globalization dynamics and boom in the oil industry that in the Middle-East in the 1970s (DoFE, 2015). Country wise status of foreign employment for the fiscal year 1993/94 up to 2016/17 (Table 2).

Table 2: Status of Foreign Employment (FY 1993/94 - 2006/07) 


\begin{tabular}{|c|c|c|c|c|c|c|c|c|c|c|c|c|c|c|c|c|c|}
\hline & $* * L I / 9$ I0Z & 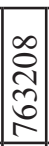 & $\begin{array}{l}- \\
n \\
\infty \\
0 \\
0 \\
\end{array}$ & ָิ & \begin{tabular}{l}
\multirow{2}{*}{} \\
gे \\
\end{tabular} & $\begin{array}{l}a \\
n \\
n \\
n \\
y\end{array}$ & $\begin{array}{l}0 \\
0 \\
\text { I } \\
\Xi \\
\end{array}$ & $\begin{array}{c}m \\
\dot{\sigma} \\
\tilde{\sigma} \\
\end{array}$ & $\underset{⿱ 乛}{\stackrel{J}{J}}$ & 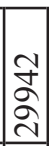 & ิㅡㄹ & $\begin{array}{l}\sqrt{\hat{0}} \\
\text { ป } \\
\end{array}$ & $\begin{array}{l}n \\
\tilde{n} \\
2\end{array}$ & $\frac{6}{6}$ & $\begin{array}{l}n \\
2 \\
2 \\
n \\
\end{array}$ & 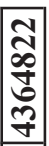 & \\
\hline & $* L I / 9$ I0Z & & \begin{tabular}{l}
\multirow{J}{0}{} \\
+ \\
+ \\
m
\end{tabular} & 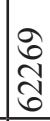 & $\begin{array}{l}\infty \\
\infty \\
\dot{\infty}\end{array}$ & $\begin{array}{l}\bar{n} \\
\tilde{n} \\
\text { v }\end{array}$ & \begin{tabular}{l}
$\infty$ \\
\multirow{b}{0}{} \\
$n$
\end{tabular} & 志 & テ & $\frac{\mathfrak{V}}{ \pm}$ & స్రి & 2 & 离 & $N$ & $\begin{array}{l}2 \\
2 \\
8 \\
+\end{array}$ & $\begin{array}{l}0 \\
0 \\
\infty \\
\infty \\
-\end{array}$ & \\
\hline & $9 \mathrm{I} / \mathrm{SIOZ}$ & & $\begin{array}{l}2 \\
\hat{2} \\
2 \\
0 \\
\end{array}$ & 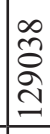 & 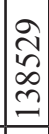 & 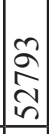 & $\underset{8}{8}$ & $\frac{0}{ \pm}$ & $\underset{\sim}{\stackrel{N}{ \pm}}$ & $\begin{array}{l}\hat{\sigma} \\
0 \\
m\end{array}$ & $\begin{array}{c}J \\
\infty \\
\infty \\
m\end{array}$ & 6 & $\frac{9}{7}$ & 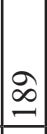 & $\begin{array}{l}0 \\
\varnothing \\
\infty \\
\end{array}$ & $\frac{m}{\frac{m}{\sigma}}$ & \\
\hline & $\varsigma \mathrm{I} / \mathrm{DI} 0 \mathrm{Z}$ & & 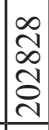 & 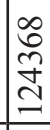 & 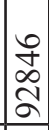 & $\begin{array}{l}2 \\
\hat{\sigma} \\
n \\
n \\
\end{array}$ & $\begin{array}{l}\infty \\
0 \\
0 \\
2 \\
2\end{array}$ & $\frac{6}{\sigma}$ & $\stackrel{\infty}{n}$ & $\begin{array}{c}8 \\
\infty \\
\text { तn }\end{array}$ & $\begin{array}{l}0 \\
\infty \\
\infty \\
N\end{array}$ & $\begin{array}{l}\infty \\
\infty \\
n\end{array}$ & 官 & 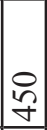 & 융 & 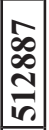 & \\
\hline & $\nabla \mathrm{I} / \mathcal{E} \mathrm{I} 0 Z$ & & 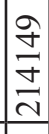 & $\begin{array}{l}\mathbb{t} \\
\infty \\
\infty \\
\\
\end{array}$ & $\begin{array}{l}0 \\
0 \\
\infty \\
0 \\
\infty \\
\end{array}$ & $\begin{array}{l}n \\
\vdots \\
\vdots \\
\dot{j} \\
\end{array}$ & $\begin{array}{l}n \\
\tilde{n} \\
2\end{array}$ & $\begin{array}{l}\infty \\
\dot{\gamma} \\
\end{array}$ & $\frac{n}{2}$ & \begin{tabular}{|l}
$n$ \\
$\stackrel{n}{5}$ \\
$m$
\end{tabular} & 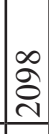 & $\equiv$ & $\begin{array}{l}n \\
0 \\
0\end{array}$ & 志 & $\begin{array}{l}\infty \\
\sim \\
2 \\
2 \\
\end{array}$ & \begin{tabular}{|l} 
\pm \\
$\infty$ \\
\multirow{2}{*}{} \\
ñ \\
\end{tabular} & \\
\hline$\stackrel{\Xi}{ \pm}$ & $\varepsilon \mathrm{I} / 乙 \mathrm{I} 0 Z$ & & 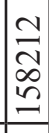 & $\begin{array}{l}0 \\
\infty \\
+ \\
0 \\
0 \\
-1\end{array}$ & مू & 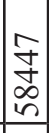 & N & $\frac{\infty}{\sim}$ & $\stackrel{\&}{\&}$ & $\frac{m}{\hat{m}}$ & 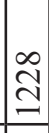 & $\begin{array}{l}2 \\
\infty \\
\text { in }\end{array}$ & $\begin{array}{l}0 \\
n \\
m\end{array}$ & 导 & $\begin{array}{l}\infty \\
\searrow \\
\end{array}$ & 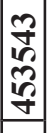 & $\frac{1}{8}$ \\
\hline 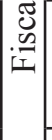 & ZI/IIOZ & & $\begin{array}{l}1 \\
6 \\
\infty \\
\infty \\
\end{array}$ & $\begin{array}{l}- \\
\infty \\
0 \\
0 \\
0\end{array}$ & \begin{tabular}{|l|}
$n$ \\
$n$ \\
0 \\
$\infty$ \\
\end{tabular} & \begin{tabular}{c}
0 \\
$\infty$ \\
\multirow{2}{*}{} \\
$n$
\end{tabular} & $\begin{array}{l}n \\
n \\
\vdots \\
\\
\end{array}$ & $\begin{array}{l}6 \\
\infty \\
\infty \\
\end{array}$ & $\begin{array}{l}\hat{\sigma} \\
n\end{array}$ & $\begin{array}{l}\hat{\sigma} \\
m\end{array}$ & $\underset{\Xi}{\Xi}$ & $\stackrel{\overbrace{}}{\sim}$ & $\begin{array}{l}\approx \\
\infty \\
\infty\end{array}$ & 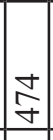 & $\begin{array}{l}6 \\
6 \\
0 \\
m \\
m\end{array}$ & \begin{tabular}{|c|} 
\\
0 \\
0 \\
0 \\
0 \\
$\infty$ \\
$e$ \\
\end{tabular} & $\frac{1}{2}$ \\
\hline & I I/0I0Z & & \begin{tabular}{l}
0 \\
$̊$ \\
2 \\
$\wp$ \\
\hdashline \\
\end{tabular} & 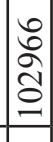 & $\underset{\cdots}{\equiv}$ & 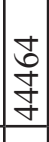 & $\frac{\infty}{n}$ & \begin{tabular}{|c|}
$\hat{y}$ \\
$\dot{\sigma}$ \\
\end{tabular} & $\underset{N}{\stackrel{\infty}{N}}$ & $\underset{\mathcal{J}}{\stackrel{\mathcal{J}}{\sim}}$ & శ̊ & $\underline{n}$ & $\begin{array}{l}n \\
\tilde{6} \\
\end{array}$ & 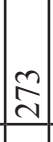 & $\begin{array}{l}\infty \\
i \\
\sim \\
\end{array}$ & 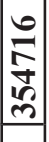 & E \\
\hline & $0 \mathrm{I} / 600 \mathrm{Z}$ & & $\begin{array}{l}\Omega \\
\infty \\
2 \\
\cdots \\
=\end{array}$ & 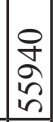 & 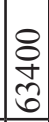 & $\begin{array}{l}\infty \\
\infty \\
m \\
m\end{array}$ & $\begin{array}{l}n \\
n \\
\infty \\
\infty\end{array}$ & $\begin{array}{l}\dot{\sim} \\
\stackrel{2}{4} \\
\dot{y}\end{array}$ & $\begin{array}{l}\stackrel{\approx}{\sim} \\
\tilde{\sim}\end{array}$ & $\begin{array}{l}n \\
\infty \\
\sim \\
n\end{array}$ & $\frac{6}{n}$ & $\begin{array}{l}\infty \\
\infty \\
\infty \\
\end{array}$ & $\stackrel{n}{\approx}$ & $\frac{0}{m}$ & ని & 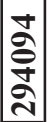 & $\begin{array}{l}\dot{0} \\
1 \\
1\end{array}$ \\
\hline & $60 / 800 z$ & & 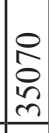 & $\begin{array}{l}n \\
2 \\
2 \\
2\end{array}$ & 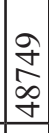 & $\begin{array}{l}\infty \\
\infty \\
0 \\
0 \\
m\end{array}$ & సิ & $\begin{array}{c}0 \\
0 \\
\text { రై } \\
\end{array}$ & 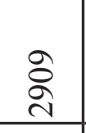 & $\underset{\stackrel{\sim}{\mathcal{J}}}{ }$ & $\widehat{N}$ & 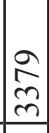 & $\begin{array}{l}\infty \\
\tilde{n} \\
n\end{array}$ & \begin{tabular}{|c|}
$\infty$ \\
$\infty$ \\
$m$ \\
$m$ \\
\end{tabular} & 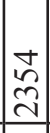 & $\begin{array}{l}\qquad \\
6 \\
2 \\
\\
\end{array}$ & 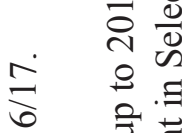 \\
\hline & $80 / L 00 Z$ & & \begin{tabular}{l}
\multirow{2}{n}{} \\
2 \\
0 \\
$n$ \\
\end{tabular} & \begin{tabular}{l}
$\mathcal{Y}$ \\
\multirow{7}{*}{} \\
2 \\
$\infty$
\end{tabular} & 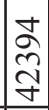 & 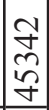 & 용 & 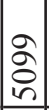 & $\stackrel{\circ}{ \pm}$ & $\begin{array}{l}0 \\
\sim \\
\tilde{N} \\
\end{array}$ & 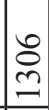 & 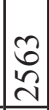 & \& & $\stackrel{0}{=}$ & $\begin{array}{l}\mathcal{O} \\
0 \\
0 \\
\end{array}$ & 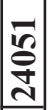 & 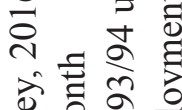 \\
\hline & 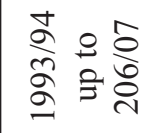 & 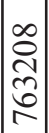 & & & & & & & & & & & & & & 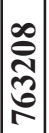 & 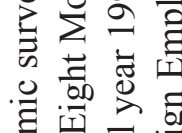 \\
\hline & 兽 & 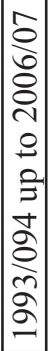 & $\frac{\substack{\pi \\
\frac{\pi}{\pi}}}{\sum_{\Sigma}^{\pi}}$ & 菍 & 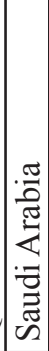 & 昰 & 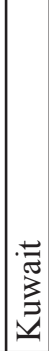 & 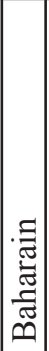 & 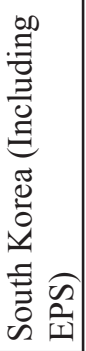 & 苂 & 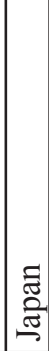 & 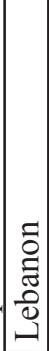 & 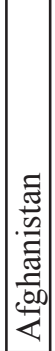 & 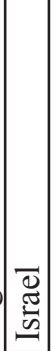 & 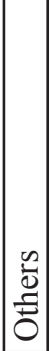 & 苞 & 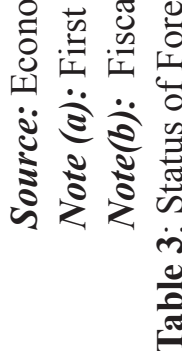 \\
\hline
\end{tabular}




\begin{tabular}{|l|r|r|}
\hline \multicolumn{1}{|c|}{ Country } & Number & \multicolumn{1}{c|}{ Percent } \\
\hline Malaysia & 1078511 & 29.94521 \\
\hline Qatar & 974239 & 27.05007 \\
\hline Saudi Arabia & 769943 & 21.37772 \\
\hline UAE & 455319 & 12.64208 \\
\hline Kuwait & 114266 & 3.172633 \\
\hline Others & 55965 & 1.553887 \\
\hline Bahrain & 43843 & 1.217315 \\
\hline South Korea & 34144 & 0.948019 \\
\hline Oman & 29942 & 0.83135 \\
\hline Japan & 17022 & 0.472621 \\
\hline Lebanon & 12671 & 0.351814 \\
\hline Afghanistan & 9573 & 0.265798 \\
\hline Israel & 6176 & 0.171479 \\
\hline
\end{tabular}

Source: Economic survey, 2016/17.

Note: $1993 / 94$ up to 2017 Feb.

Figure 1: Situation of Countrywise Foreign Employment of Nepal

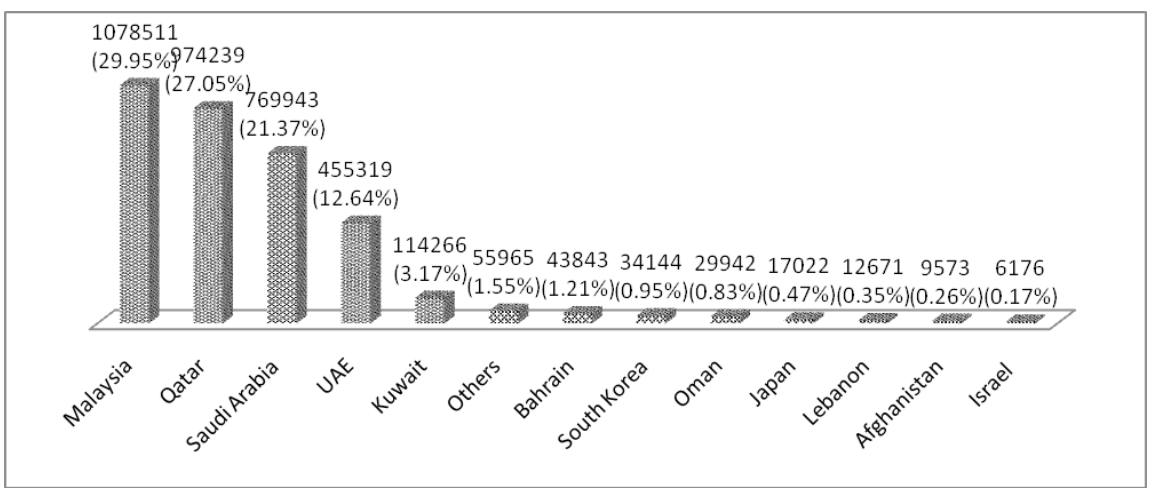

Source: Economic survey, 2016/17.

Nepalese to foreign employment was estimated more than 4.36 million, except India, outflow of Nepalese labour to major destination countries were more than nine such as Saudi Arabia, Qatar, UAE, Bahrain, Oman, Malaysia, Korea etc. Among all destination countries Malaysia, Qatar, Saudi Arabia, UAE and Kuwait were top five countries. In addition USA, UK, EU and Japan were minor destinations. India was traditional destination of unskilled labour.

Table 4: Remittance Inflow and Contribution on GDP 


\begin{tabular}{|c|r|r|r|}
\hline $\begin{array}{c}\text { Fiscal } \\
\text { Year }\end{array}$ & $\begin{array}{c}\text { Remittance } \\
\text { in flow (in Rs. } \\
\text { Billions) }\end{array}$ & $\begin{array}{c}\text { Contribution } \\
\text { in GDP (in \%) }\end{array}$ & $\begin{array}{c}\text { Share of remittance } \\
\text { on convertible foreign } \\
\text { exchange (in\%) }\end{array}$ \\
\hline $2007 / 08$ & 142.7 & 17.5 & 58.84 \\
\hline $2008 / 09$ & 209.7 & 21.2 & 59.87 \\
\hline $2009 / 10$ & 231.7 & 19.4 & 61.52 \\
\hline $2010 / 11$ & 253.6 & 18.5 & 59.4 \\
\hline $2011 / 12$ & 359.6 & 23.5 & 62.09 \\
\hline $2012 / 13$ & 434.6 & 25.6 & 62.63 \\
\hline $2013 / 14$ & 543.3 & 27.7 & 63.61 \\
\hline $2014 / 15$ & 617.3 & 29 & 61.92 \\
\hline $2015 / 16$ & 665.1 & 29.6 & 64.5 \\
\hline $2016 / 17^{*}$ & 699 & 26.9 & 66.46 \\
\hline
\end{tabular}

Source: Economic survey, 2016/17.

Note: First $8^{\text {th }}$ Months

Figure 2 (B): Trends of Remittance (2008/09 - 2016/17)

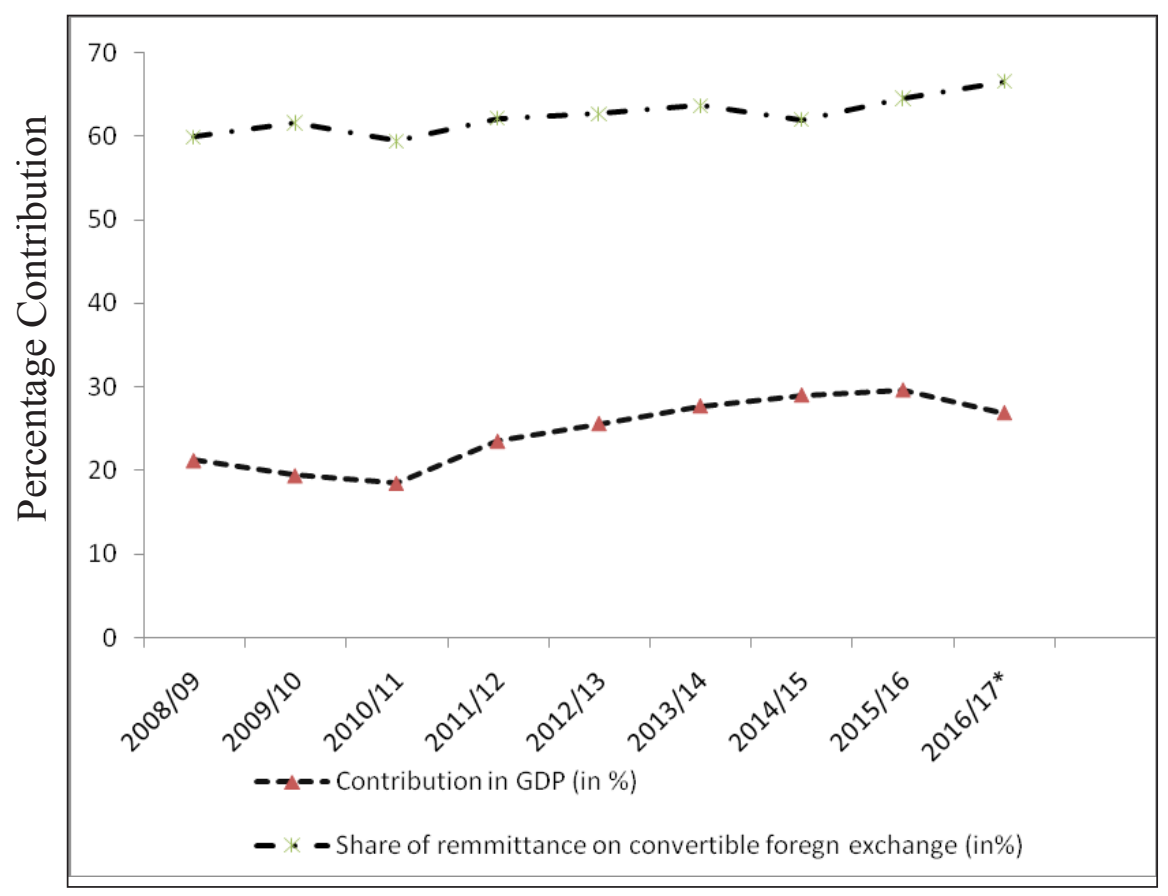

Source: Based on Table 3. 
Migration has become an important part of the Nepalese labour market at present. Labour migration to India and rural to urban are very common phenomenon. The number of foreign employment bound workers, excluding India was reached close to 3.5 million in early 2014 and on are average of 1500 workers continue to leave the country legally each day (MoF, 2014). Remittance inflow was seemed to be continued to grow along with the increase in the number of labour migrants. Remittance income was reached USD 5.946 billion (29.6 of GDP) in fiscal year 2072/73(Economic Survey, 2074). It was estimated that remittance inflow in fiscal year 2016/17 (first Eight Months) was Rs. 699 billion and it was 26.9 percent contribution in GDP and 66.46 percent of convertible foreign exchange (MoF, 2014).

According to the Living Standard Survey (2011) 56 percent of household had received remittances both from domestic and international sources in 2010/11. Similar figure was only 23 percent in 1995/96 increasing with only 20 percent coming from Nepal in 2010/11 vs 45 percent in fiscal year 1995/96. About 33 percent of households were found to be at least one member living abroad, equivalent to 8.7 percent of the population (CBS, 2011). In total foreign employment share of Malaysia, Qatar, Saudi Arabia and U.A.E was approximately 94 percent. Outflow of labour in major destination was dominated by unskilled labour, although policy was to encourage the outflow of skilled labour so that higher money wage could be drawn. Wage income of unskilled labour was unexpectedly lower than started wage income. In India, there was outflow of unskilled labour larger than in major destination.

A nationwide migration survey conducted in 2009, Nepal migration survey 2009, estimated that 41 percent of all the migrant workers were working in India, 38 percent in the Gulf countries, 12 percent in Malaysia and 8.7 percent in developed countries (World Bank, 2011). The data provided by the Department of Foreign Employment (DoFE) also corroborate the finding of the survey, excluding India. It shows that between 1993/94 and 2011/12 the cumulative share of Malaysia was 31.4 percent, the gulf countries ( Qatar, Saudi Arab, UAE, Kuwait, Bahrain and Oman) was 65.3 percent and other countries constituted a minuscule share of 3.3 percent (DoFE, 2015 ). Most of the migrants were working in low paying job more than one-tenth of the migrant to India were working in agriculture and 62 percent of migrants in Malaysia and about 27 percent in gulf countries were working in manufacturing sectors.

The number of migrant's workers, their level of earning and the sending rate determine remittance inflows. Almost three quarters of the migrants had delivered remittance to their homes. The sending rate of migrants in Malaysia was the highest (78.8\%) followed by India (73.3\%) and 
the others $(71.1 \%)$. The amount of remittance received differed by migrant's destination. Households with the migrant in the gulf received of Rs.163, 000/ year (2120US), in Malaysia Rs 113,000 (1470 US) and in India Rs 62000 (800 US) whereas household within migrants in other developed countries, received a much higher amount (World Bank, 2011). Almost half of the remittance came from the gulf countries, however, in terms of the remittance single source country, India was the largest source of remittance but its role in the total figure was found to be declining over the period (Sharma, 2013).

At the household level, about 56 percent of the households received remittances in 2010/11, both from internal as well as external sources. The share of external remittance in total remittances was 81.4 percent, a substantial increase from 76.5 percent in 2003/04 and 55.3 percent in 1995/96. The nominal average amount of remittance received by a household was Rs. 80436 and constituted about 31 percent of the income among remittance receiving household (CBS, 2011). Most of the remittance receiving household had used it for daily consumption purposes and for paying loans. Nepal Living Standard Survey (III) shows that 78.8 percent of the total remittance received by the house hold was used for daily consumption and 7.1 percent was used for loan repayments. The use for capital formation was 2.4 percent and 0.5 percent was used for business purposes. Other uses were to acquire household property $(4.5 \%)$ and to educate children $(3.5 \%)$ and the remaining $(2.5 \%)$ was used for another purposes. Remittance was typically spent on land and housing purchases. These were safe investment for the households, but in macroeconomic terms, these were non-productive assets, with no lasting impact on the country's real income. Remittance is outcome of foreign employment. There was positive correlation between growth and quality and quantity of outflow of labour. Growth of remittance strengthens expansion of local and national economic activities.

The inflow of remittance in Nepal seemed to be expansionary. In 2017, it was estimated Rs.665.1 billion. In 2013 it was Rs 266 billion slightly declined from 360 billion in 2012 but inclined from 228 billion in 2010. In 2008, remittance was Rs 75 billion. In accordance with Nepal Rastra Bank (NRB) it was only of 40 percent out flowing labour. Its share was 50 percent in total foreign exchange and 14 percent of GDP in 2008 and 23 percent of GDP in 2013. At present its share to GDP is about 29.6 percent and its share in foreign reserve is estimated to be 78.9 percent. At the household level, remittance helps to smoothen consumption and investment in human and physical capital. It also generates benefits to the community, through the expenses on locally produced goods and services, and helps poverty reduction since the money has been utilized for rural development. The IMF country report states that, in Nepal's case, the penetration of the remittances into the remote villages had 
helped in poverty alleviation and in the absence of remittance the present level of poverty would be 36 to 37 percent (IMF, 2006). In indicates that remittance and poverty reduction were inversely correlated.

Remittance contributes substantially to foreign exchange reserve and helps to finance Nepal's burgeoning imports. Empirical studies have shown that the increase inflow of remittances has contributed between one third to one half of the overall reduction of poverty in the country, which went down by all 11 percent points between 1995/96 and 2003/04 (Lokshin, Osmoloviski \& Glinskaya, 2010, World Bank 2006). Between 2003/04 and 2010/11 high remittance inflow had been attributed as the main cause behind the decline in absolute poverty to 25.16 percent in 2010/11 from 30.80 in 2003/04 (ADB, 2012 NIDS and NCCR 2012).

Similarly, the decline inequality (Gini Coefficient) to 32.94 from 41.40 between 2010/11 and 2003/04 was due to the more than proportionate increase in expenditure (income) capacity of the poor because of increased remittance(or income) capacity of the poor because of increased remittance inflows (Sharma, 2013). In addition labour migration had also made a considerable contribution in reducing under employment. Other positive effects include higher wages resulting from reduced labour supply and better rental terms for farming. A pernicious effect of huge-flow of remittance in Nepal pertains to symptoms of the so called Dutch diseasessignificant resources allocation effects of remittances on the economy that reduces its competitiveness (Sharma, 2013, World Bank 2011). There was an appreciation of the real exchange rate and the tradable sector had shrunk in relation to the non-tradeable sector. In addition, the wage rate was also risen due to increased demand of labour in the non-tradable sector and the shortage of workers due to migration (Sharma, 2013).

As most of the remittance was being used for household consumption it was fuelled aggregate demand to higher level, much more than the economy's output and productivity would permit. Because of declining agriculture and manufacturing sectors, the increased demand had been fulfilled through imports thus widening the trade deficit further. However, the increasing imports had positively contributed to government revenues. Remittances had also contributed to demand-push inflation and occasionally excess liquidity.

The formal and organized channel of remittance was still few because of lack of awareness and transfer cost. However, informal and unorganized channel was quite popular because of least transfer cost although its financial security and liability were said critical. Therefore, actual remittance is a subject to be examined. 


\section{CONCLUSION}

The decade long conflict hit hard the economy. Remittance income had saved the country from fiscal imbalances. Given major contribution of remittances, it is imperative to systematize the foreign migration of workers, flow of remittances and their productive investment. Nepalese economy is heavily dependent on remittance income sent by migrant workers at present. This seems to increase the vulnerability of the economy since any disruption in remittance would create havoc in the economy. The government of Nepal seems concentrated on attracting remittances but paying low attention to mitigating the adverse effects and promoting it towards productive uses.

In Nepalese economy labour force is rapidly growing. More than 450 thousand youths enter the labour market annually. But lack of productive job availability in the country, migration has become an important part of the Nepalese labour market. Hence remittances have become the largest sources of foreign exchange, substantially contributing to foreign exchange reserves and helping to finance to rapidly increasing imports. It has also contributed to reduce poverty to some extent. However, the major portion of the earning has been used for daily consumption and insignificant portion is going to productive investment. Remittances are beneficial at household and community level however it cannot help for sustainable development without its strategic management. Formulation and implementation of pro poor migration policy is the need of today. The government should play proactive role to promote foreign employment by inducing and adhering to the policy of economic diplomacy.

\section{REFERENCES}

Asian Development Bank (ADB) (2012) : Food security and poverty in Asia and Pacific key challenges and policy issues. Manila: Asian Development Bank.

Central Bureau of Statistics (CBS), (2009). Nepal labour force survey 2008. Kathmandu : CBS.

- - - (2011). Poverty in Nepal in 2010/11. Kathmandu : CBS.

- - - (2014). Annual statistics, Kathmandu: National Planning Commission.

Department of Foreign Employment (DoFE) (2015). Labour migration for employment: A status report for Nepal: 2013/2014, Kathmandu: Ministry of Labour and Employment.

IMF (2005). Nepal poverty reduction strategy paper, annual progress report. IMF Country Report No. 06/433. 
Islam, Rizwanul (2014). Addressing the employment challenge through the sectoral pattern of growth. Kathmandu: International Labour Office.

Karna, S.K. (2017), Political economy of labour migration and remittances in nepal. in political economy of Nepal. Kathmandu: Central Department of Economics (TU), and Friedrich Ebert Siftung.

Lokshin, M. M. Bontch-Osmolovski \& Glinskaya E. (2010). Work related migration and poverty reduction in Nepal. Review of Development Economics 14(2): 323-332.

MoF (2014). Budget speech 2017/18.Kathmandu : Ministry of Finance.

- - (2017). Economic survey - Kathmandu : Ministry of Finance.

NIDS \& NCCR (2012). Nepal migration year book 2011. Kathmandu: Nepal Institute for Development Studies (NIDS) and National Center of Competency in Research North-South (NCCR).

NPC (2017). Three year fourteenth plan, 2017/18-2018/19. Kathmandu: National Planning Commission.

- - (2013). Three year interim development plan, 2010/11-2012/13. National Planning Commission, Government of Nepal.

- - (2014). Nepal human development report. Kathmandu: National Planning Commission.

- - (2016). Nepal and the millennium development goals, final status report 2000-2015. Kathmandu : National Planning Commission.

NRB/GON. (2017). Monetary policy for FY 2016.17. Retrieved from nrb. org.np.

Pyakuryal, B. (2013), Nepal's development tragedy: Threats and possibilities. Kathmandu: Fine Prints.

- - (Ed.)(2015). Macro Economics, A radical rethinking in growth, inequality and inclusiveness in Nepal. Kathmandu: Mandala Book Point.

Sharma, N. K. (2013). Economics of Nepal. Kathmandu: Pairavi Prakashan.

Shrestha, P.K.(2017). Nepal's experience of globalization and future prospect of its economy. In political economy of Nepal. Kathmandu: Central Department of Economics (TU) and Friendrich Ebert Stiftung.

Sijapati, B. (2014). Enhancing employment-centric growth in Nepal: Situational analysis for the employment policy. Kathmandu: International Labour Office.

World Bank (2011). Large scale migration and remittance in Nepal: Issues, challenges and opportunities. Washington DC: World Bank. 